Research Paper

\title{
Co-overexpression of RIOK1 and AKT1 as a prognostic risk factor in glioma
}

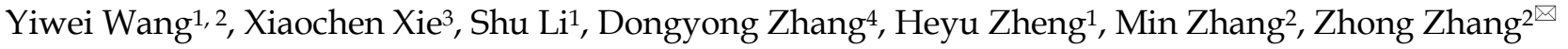 \\ 1. Department of Human Anatomy, Shenyang Medical College, Shenyang City, Liaoning Province 110034, P.R. China. \\ 2. Department of Pathology, College of Basic Medical Sciences, Shenyang Medical College, Shenyang City, Liaoning Province 110034, P.R. China. \\ 3. Department of Endocrinology and Metabolism, Institute of Endocrinology, Liaoning Provincial Key Laboratory of Endocrine Diseases, The First Affiliated \\ Hospital of China Medical University, China Medical University, Shenyang, Liaoning, 110001, P.R. China. \\ 4. Department of Neurosurgery, First Affiliated Hospital of China Medical University, Heping District, Shenyang City, Liaoning Province, 110001, P.R. China \\ $\triangle$ Corresponding author: E-mail: zhangzhong369@symc.edu.cn
}

( ) The author(s). This is an open access article distributed under the terms of the Creative Commons Attribution License (https://creativecommons.org/licenses/by/4.0/). See http://ivyspring.com/terms for full terms and conditions.

Received: 2021.03.18; Accepted: 2021.07.19; Published: 2021.07.25

\begin{abstract}
Glioblastoma multiforme (GBM) is one of the most frequent primary malignancies of the brain. Although the treatment strategy has significantly improved, patient prognosis remains poor. In vitro studies have shown that the right open reading frame kinase 1/protein kinase B (RIOK1-AKT) signaling pathway plays an important role in the malignant phenotype of glioma cells. This study aimed to investigate the co-expression of RIOKI and ATK in glioma tissues and its clinical significance. Compared with normal tissues, RIOK 1 and AKTI expression were significantly upregulated in glioma tissues. In addition, patients with higher World Health Organization staging grades had increased RIOK 1 and AKT1 expression levels, and RIOK1 and AKT1 expression were positively correlated. Notably, both RIOK1 and AKT1 expressions were correlated with poor prognosis. In vitro experiments showed that silencing RIOKI inhibited the proliferation, migration, and invasion of glioma cell lines by suppressing AKT and c-Myc expression. These results indicate that the RIOK1-AKTI axis could play an important role in GBM progression.
\end{abstract}

Key words: RIOK1, AKT1, c-Myc, GBM

\section{Introduction}

Glioblastoma multiforme (GBM) is one of the most frequent primary malignancies of the brain, accounting for $60 \%$ of primary intracranial tumors and $80 \%$ of the primary intracranial malignancies [1, 2]. Despite advances in surgery, radiotherapy and chemotherapy technology, patient prognosis remains poor, especially for those with high-grade disease [3-6]. It is therefore important to explore potential molecular mechanisms that promote the malignant progression of GBM, which might identify new treatment strategies.

The right open reading frame kinase (Rio kinase) family consists of three members (RIOK1, RIOK2 and RIOK3) that are involved in ribosome synthesis and processing in vivo $[7,8]$. RIOK2 expression is increased in non-small cell carcinomas such as lung squamous cell carcinoma and adenocarcinoma and is associated with the degree of differentiation and pathological stage $[9,10]$. RIOK3 can also promote the proliferation, invasion and migration of glioma cells through protein kinase $\mathrm{B} /$ mammalian target of rapamycin (AKT/mTOR) signaling pathway [11]. RIOK1 is an important member of the atypical serine/threonine kinase family. A previous study demonstrated that RIOK1 is highly expressed in colorectal cancer cells and promotes proliferation and invasion by activating the nuclear factor- $\mathrm{kB}$ signaling pathway of Ras mutant tumor cells, but it has no effect on Ras wild-type cells [10]. In addition, silencing RIOK1 suppresses the proliferation, migration, and invasion of breast, lung and colon cancer cell liens [9]. RIOK1 and RIOK2 are also highly expressed in glioma cell lines; overexpressing RIOK1/2 contributes to proliferation, while their knockdown induces 
apoptosis and renders cells more sensitive to chemotherapy [12]. AKT1 is an important part of the AKT signaling pathway, and its upregulation is associated with poor prognosis in patients with GBM [13]. In a number of tumor types, increased AKT1 activity can promote tumorigenesis and development [14-16]. RIOK1 plays important roles in many kinds of tumors, but its expression in different types of glioma cells and its effect on biological behavior are still unclear. It is therefore essential to investigate RIOK1 and AKT1 expression in glioma to identify new therapeutic approaches and prognostic biomarkers.

In this study, we confirmed that RIOK1 and AKT1 levels significantly increased in GBM tissues and were positively associated with tumor malignancy, while knockdown of RIOK1 inhibits glioma cell growth through AKT1 and c-Myc in vitro. Our results highlight RIOK1 as a novel therapeutic target in GBM.

\section{Materials and Methods}

\section{Gene expression analysis}

RIOK1 and AKT1 mRNA expression data were obtained from The Cancer Genome Atlas (TCGA) GBMLGG (689 gliomas) and GTEX (1157 normal tissues) datasets. We obtained the scatter diagram of RIOK1 and AKT1 expression correlation from TCGA GBMLGG (https://portal.gdc.cancer.gov/). RNAseq data was given in TPM (transcripts per million reads) format, and expression levels compared between samples after $\log 2$ transformation.

\section{Patients and tissue specimens}

A total of 106 glioma tissue samples were collected by the Department of Neurosurgery of the First Affiliated Hospital of China Medical University from 2012 to 2018. Patient clinicopathological features are presented in Table 1. Ten non-tumor brain tissue samples from patients with traumatic brain injuries who underwent internal decompression served as the control group. Glioma samples were classified as grade I, II, III, or IV based on the World Health Organization (WHO) guidelines. This study was approved by the Ethical Committee of First Affiliated Hospital of China Medical University. Written informed consent was obtained from participants.

\section{Tissue microarray and immunohistochemistry (IHC)}

All tissue specimens were fixed in $4 \%$ paraformaldehyde, embedded in paraffin, then dehydrated in an alcohol gradient and xylene until they were transparent. Paraffin-embedded tissue samples were used to construct a tissue microarray using the Manual Tissue Microarrayer (Quick-ray,
UniTMA, Seoul, South Korea). IHC was performed on tissue microarray samples using routine experimental procedures [17]. The primary antibodies were rabbit anti-RIOK1 (N-terminal) polyclonal antibody (1:100; ab176005, Abcam, Cambridge, UK) and AKT1 polyclonal antibody (1:100; ab194201, Abcam). An UltraSensitive $^{\mathrm{TM}}$ SP (Mouse/Rabbit) IHC Kit (KIT-9710, Maixin, Shenzhen, China) was used to label tissue. Scoring criteria for IHC were described in our previous publication [17].

Table 1. Clinicopathological characteristics and RIOK1/AKTI expression in 106 glioma patients

\begin{tabular}{|c|c|c|c|c|c|c|c|}
\hline \multirow[t]{2}{*}{ Variables } & \multirow{2}{*}{$\begin{array}{l}\text { Number } \\
\text { of cases }\end{array}$} & \multicolumn{2}{|c|}{ RIOK1 Expression } & \multirow[t]{2}{*}{$p$-value } & \multicolumn{2}{|c|}{ AKT1 Expression } & \multirow[t]{2}{*}{$p$-value } \\
\hline & & High & Low & & High & Low & \\
\hline \multicolumn{8}{|l|}{$\begin{array}{l}\text { Ages } \\
\text { (years) }\end{array}$} \\
\hline$<50$ & 37 & 24 & 13 & 0.686 & 23 & 14 & 0.324 \\
\hline$\geq 50$ & 69 & 42 & 27 & & 36 & 33 & \\
\hline \multicolumn{8}{|l|}{ Gender } \\
\hline Male & 49 & 34 & 15 & 0.161 & 30 & 19 & 0.285 \\
\hline Female & 57 & 32 & 25 & & 29 & 28 & \\
\hline \multicolumn{8}{|l|}{$\begin{array}{l}\text { Tumor } \\
\text { size }(\mathrm{cm})\end{array}$} \\
\hline$<3$ & 35 & 18 & 17 & 0.106 & 13 & 22 & $0.007^{* *}$ \\
\hline$\geq 3$ & 71 & 48 & 23 & & 46 & 25 & \\
\hline \multicolumn{8}{|l|}{ KPS } \\
\hline$<80$ & 62 & 40 & 22 & 0.570 & 35 & 27 & 0.846 \\
\hline$\geq 80$ & 44 & 26 & 18 & & 24 & 20 & \\
\hline \multicolumn{8}{|l|}{ WHO } \\
\hline I & 3 & 2 & 1 & $0.008^{* *}$ & 1 & 2 & $0.022^{*}$ \\
\hline II & 31 & 12 & 19 & & 10 & 21 & \\
\hline III & 26 & 17 & 9 & & 17 & 9 & \\
\hline IV & 46 & 35 & 11 & & 31 & 15 & \\
\hline \multicolumn{8}{|l|}{$\begin{array}{l}\text { Survival } \\
\text { state }\end{array}$} \\
\hline Alive & 40 & 18 & 22 & $0.004^{\star *}$ & 17 & 23 & $0.034^{*}$ \\
\hline Death & 66 & 48 & 18 & & 42 & 24 & \\
\hline
\end{tabular}

\section{Cell culture and transfection}

Glioma cell lines U87, U251, U373, U118, and A172 were purchased from the Cell Bank of the Chinese Academy of Sciences (Shanghai, China). All cells were cultured in Dulbecco's modified Eagle medium (Thermo Fisher Scientific, Waltham, MA, USA) supplemented with $10 \%$ fetal bovine serum (FBS) (HyClone, Logan, UT, USA) and maintained in standard conditions $\left(5 \% \mathrm{CO}_{2}\right.$ and $95 \%$ atmosphere, $37^{\circ} \mathrm{C}$ ). The U251 and U87 cell lines were transfected with RIOK1-RNAi-lentivirus from GeneChem Company (Shanghai, China) and screened by puromycin. The shRNA control sequence was 5'-UUCUCCGAACGUGUCACGUtt-3'; the shRNA RIOK1 sequence\#1 was 5'-GTCATGAGTTTCATC GGTAAA-3'; and the shRNA RIOK1 sequence\#2 was 5'- GGCAAATAGAATGAGAACCAT-3'.

\section{Immunoblot analysis}

Cells and tissues were scraped in radio- 
immunoprecipitation assay buffer containing 1\% phenylmethylsulfonyl fluoride and phosphorylase inhibitor to obtain total protein samples. The concentration was measured by a BCA kit (P0010, Beyotime, Shanghai, China). Samples ( $20 \mu \mathrm{g}$ protein) were subjected to $10 \%$ sodium dodecyl sulfatepolyacrylamide gel electrophoresis and transferred to polyvinylidene fluoride membranes. After blocking with evaporated milk, the membranes were incubated with primary antibodies: RIOK1 (N-terminal) polyclonal antibody (1:1000; ab176005, Abcam), AKT1 polyclonal antibody (1:1000; ab194201, Abcam), c-Myc polyclonal antibody (1:1000; ab32072, Abcam, Cambridge, UK), glyceraldehyde 3-phosphate dehydrogenase monoclonal antibody (1:5000; 60004-1-lg, Proteintech, Wuhan, China). The blots were then labeled with appropriate secondary antibodies and visualized by enhanced chemiluminescence.

\section{MTT cell viability assay}

Cells were seeded in 6-well plates at a density of 3000 per well. The medium was exchanged for MTT (3-(4,5-dimethylthiazol-2-yl)-2,5-diphenyltetrazolium bromide) (Sigma-Aldrich, St. Louis, MO, U.S.) solution at $0,24,48$, or $72 \mathrm{~h}$. After 2-h incubation, the MTT solution was exchanged for dimethyl sulfoxide, and the absorbance of each well was measured at $490 \mathrm{~nm}$.

\section{Colony formation assay}

Cells were seeded in 6-well plates (500-1000 cells/well) and cultured for 13 days. Cells were fixed with $4 \%$ paraformaldehyde and stained with crystal violet (Solarbio Science \& Technology Co., Ltd. Beijing, China). Plates were imaged, and colonies were counted and statistically analyzed.

\section{Transwell assay}

Cell invasion and migration abilities were evaluated with Transwell assays. Briefly, $1-3 \times 10^{4}$ cells/200 $\mu \mathrm{l}$ (FBS-free medium) were seeded into the upper Transwell chamber (Corning, Corning, NY, USA), and 10\% FBS medium was added to the lower chamber. After $24 \mathrm{~h}$ of culture, the bottom membrane of the chamber was fixed with $4 \%$ paraformaldehyde and stained with crystal violet. The cells were counted and imaged with an optical microscope (D-35578, Leica, Wetzlar, Germany). For invasion assays, the upper chambers were coated with Matrigel (BD Biosciences, Franklin Lakes, New Jersey, USA).

\section{Statistical analysis}

All the results were analyzed by SPSS software (IBM SPSS Statistics 21, IBM Corp., Armonk, NY, USA). Correlations between RIOK1/AKT1 expression and clinicopathological characteristics were analyzed by Pearson $\chi^{2}$ test. The overall survival (OS) curves were plotted with the Kaplan-Meier method and analyzed by log-rank tests. Three or more groups were compared by one-way analyses of variance. Differential RIOK1/AKT1 mRNA expression levels were analyzed with Mann-Whitney U tests. Spearman correlation analysis was performed to assess the relationship between RIOK1 and AKT1.

\section{Results}

\section{RIOKI and AKT1 overexpression in glioma patient tissues}

RIOK1 and AKT1 mRNA levels were significantly higher in glioma patient tissues compared to normal brain tissues (TCGA-GBMLGG and GTEx databases, Figure 1A), and their expression levels were significantly correlated (TCGA-GBMLGG databases, $p<0.001, r=0.440$, Figure 1B). RIOK1 and AKT1 protein levels were determined by IHC in 106 glioma and 10 control samples using tissue microarrays. RIOK and AKT1 staining intensity were weak in normal tissues but increased in glioma samples in a WHO grade-dependent fashion (Figure 1C). We performed confirmatory western blots using 6 control samples and 15 low-grade (WHO I-WHO II) and 15 high-grade (WHO III-WHO IV) glioma tissues. The results showed that RIOK1 and AKT1 protein levels were upregulated in glioma, with the highest expression in high-grade samples (Figure 1D-G and Supplementary Figure 1). Correlation analysis of the western blot data showed that RIOK1 and AKT1 expression were significantly correlated in glioma samples $(p<0.001, \mathrm{r}=0.7084$, Figure 1E).

\section{Association of RIOKI and AKT1 expression with clinicopathological characteristics and survival in glioma patients}

We analyzed the associations between clinicopathological characteristics and of RIOK1 and AKT1 expression in 106 patients with GBM. RIOK1 expression was significantly associated with WHO grade $(p=0.008)$ and OS $(p=0.004)$. There were no significant differences for other variables including age, sex, tumor size, or Karnofsky performance status scores $(p>0.05$, Table 1). AKT1 expression was related to tumor size $(p=0.007)$, WHO grade $(p=0.022)$ and OS $(p=0.034)$. Spearman's correlation analyses showed that the low co-expression rate of RIOK1 and AKT1 was $70.21 \%(33 / 106)$ and the high co-expression rate of RIOK1 and AKT1 was $88.14 \%(52 / 106, p<0.001)$ (Table 2). These results confirm RIOK1 and AKT1 expression are positively correlated in GBM tissue. The OS rate suggests that patients with high RIOK1 and AKT1 expression are more likely to have a poor prognosis (Figure 2). 
Table 2. Correlation between expression of RIOK1 and AKT1 in glioma patients

\begin{tabular}{|c|c|c|c|c|c|}
\hline & \multirow[t]{2}{*}{ Tissues } & \multicolumn{4}{|c|}{ Expression of RIOK1 } \\
\hline & & Low (\%) & High (\%) & $\mathbf{x}^{2 \text {-value }}$ & $p$-value \\
\hline \multirow[t]{2}{*}{ Expression of AKT1 } & Low Expression (\%) & $33(70.21)$ & $14(29.79)$ & 37.906 & $<0.001$ \\
\hline & High Expression (\%) & $7(11.86)$ & $52(88.14)$ & & \\
\hline
\end{tabular}

A

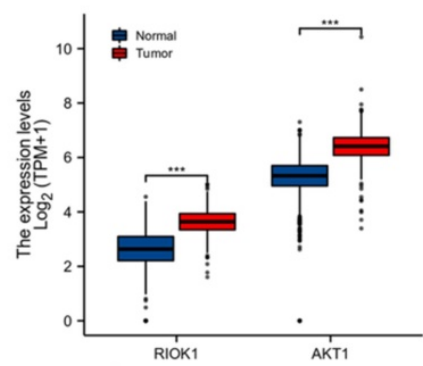

B

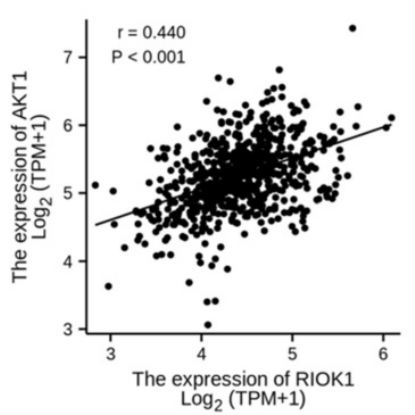

C

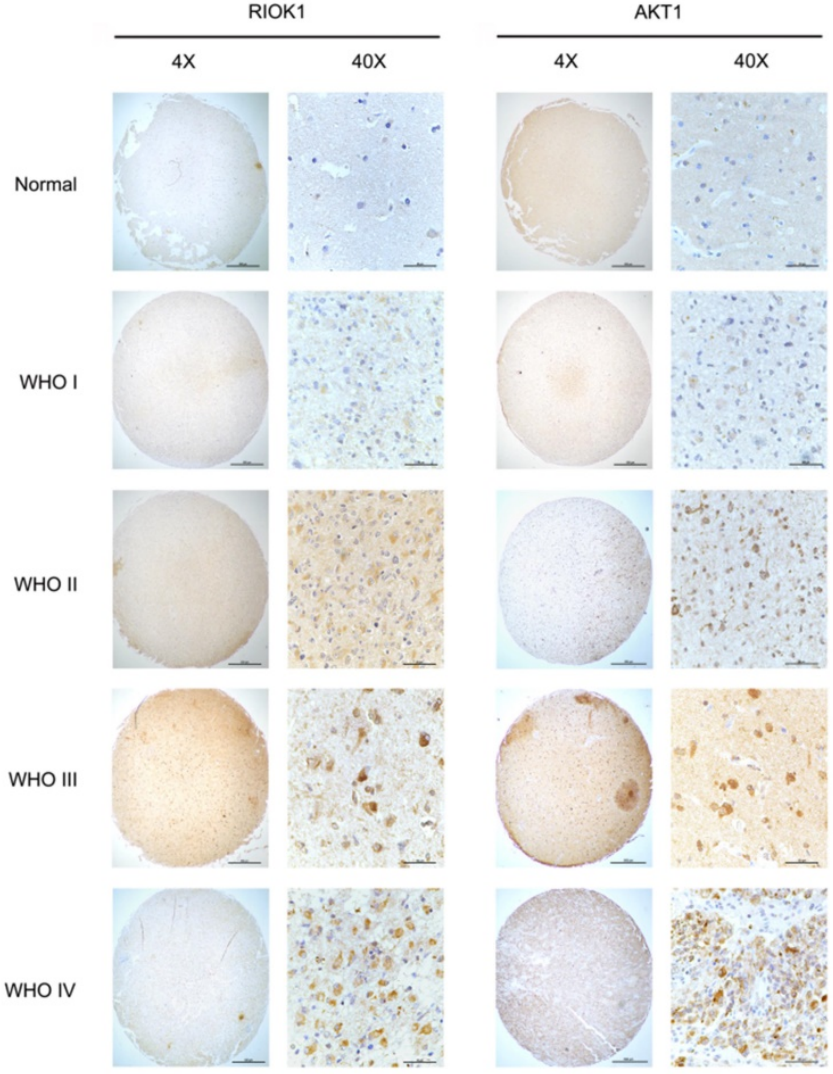

D

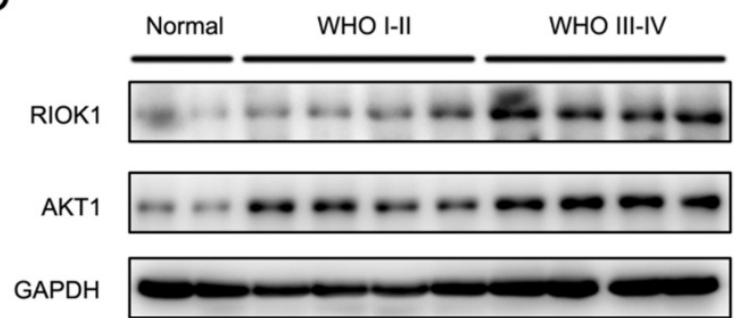

F

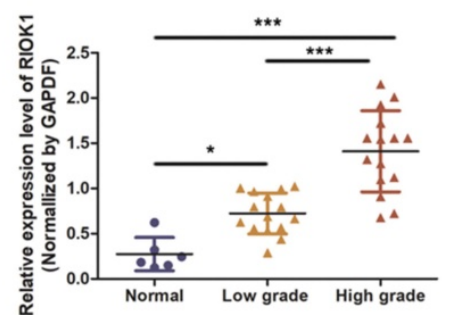

E

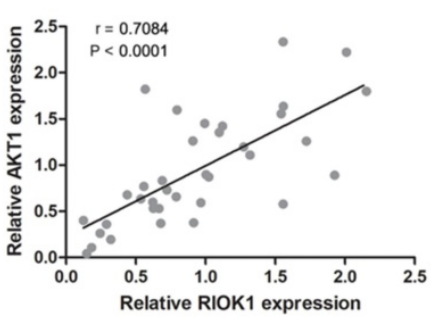

G

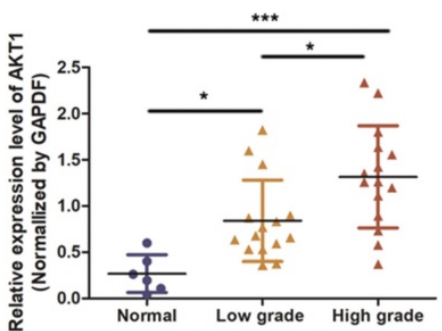

Figure 1. The expression of RIOK1 and AKT1 were up-regulated in glioma tissues and correlated with the prognosis of glioma patients. A. Data from TCGA and GTEx (RNA-seq; Normal, $n=1152$; glioma, $n=689$ ) showed that RIOK1/AKT1 were increased in glioma tissues compared with normal brain tissues (*** $p<0.001$ ). $B$. Correlation between RIOK1 and AKT1 expression in TCGA glioma $(r=0.440, p<0.001)$. C. Relative expression of RIOK1 and AKT1 in glioma and normal tissues through IHC. D. RIOK1 and AKT1 expression in Western blot. E. Correlation between RIOK1 and AKT1 expression $(r=0.7084, p<0.001)$. F, G. Relative protein expression of RIOK1 and AKT1 in 6 normal tissues and 30 glioma tissues $(* p<0.05, * * * 00,001)$. 
A

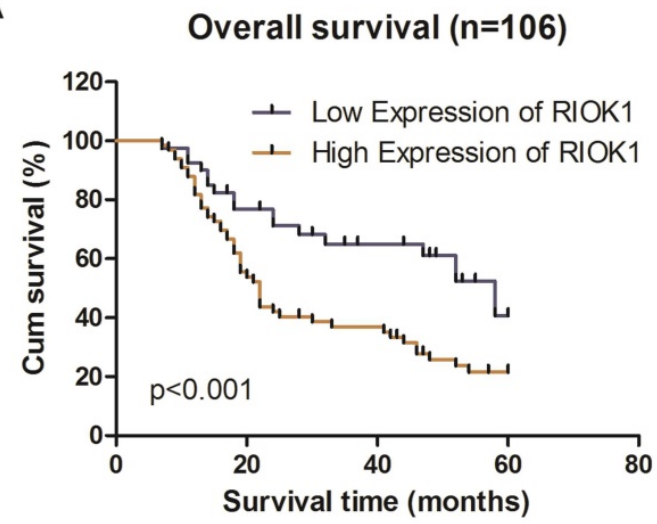

B

Overall survival $(n=106)$

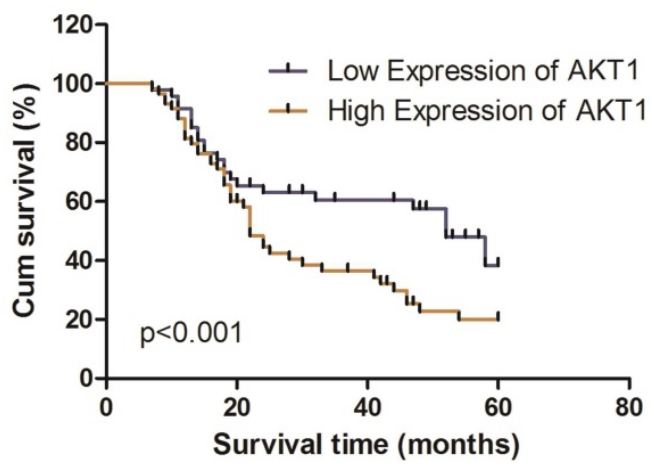

Figure 2. Kaplan-Meier survival analysis according to RIOK1 and AKTI protein expression in patients with glioma. A. Overall survival between the high RIOK1 group and Low RIOK1 group. B. Overall survival between the high AKT1 group and Low AKT1 group.

A

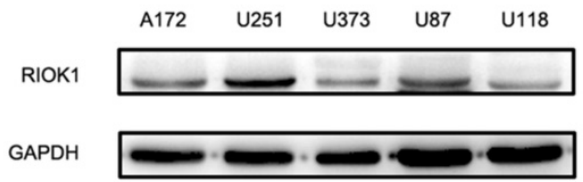

C

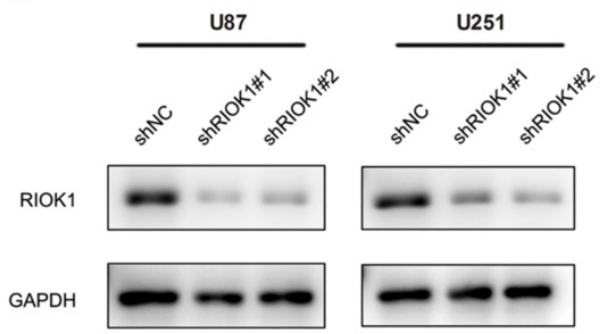

B

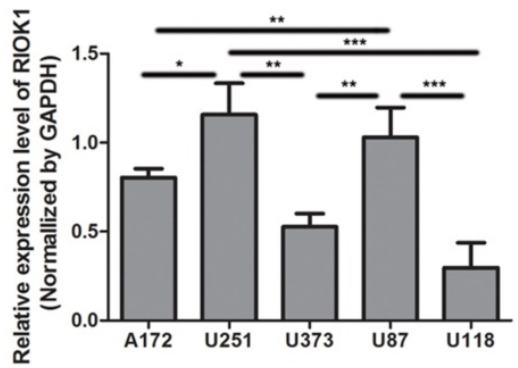

D

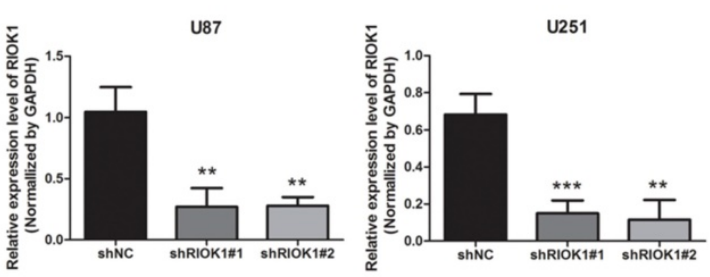

Figure 3. RIOK1 expression in glioma cells and silencing RIOK1 in U87 and U251 cells. A. The protein expression of RIOK1 in human cell lines (A172, U251, U373, U87 and U1 18). B. Gray value analysis of RIOK1 expression in glioma cell lines, ${ }^{*} p<0.05,{ }^{*} p<0.01$, $* * * p<0.01$. C. The transfected efficiency of shRIOK $1 \# 1$ and shRIOK $1 \# 2$ was detected by Western blot. D. The statistical diagram of RIOK1 protein expression in each shRNA groups, ${ }^{* *} p<0.01, * * * p<0.01$.

\section{RIOKI expression in glioma cell lines and RIOK1 stable silent cell line construction}

RIOK1 protein expression in tumor cell lines was analyzed by western blot, which showed higher levels in U251 and U87 cell lines compared to A172, U373, and U118 cell lines (Figure 3A and B). Therefore, we silenced RIOK1 expression in U87 and U251 cell lines to clarify the function of RIOK1 in glioma cells. To avoid off-target effects, we selected two shRNAs (shRIOK1\#1, shRIOK1\#2) for transfection, and efficiency was detected by Western blot (Figure 3C and D).

\section{RIOK1 knockdown inhibits glioma cell proliferation, migration, and invasion through AKTI and c-Myc}

To evaluate the roles of RIOK1 in glioma cells, we first assessed the effect of silencing RIOK1 on glioma cell proliferation (U87 and U251). According to the MTT growth curves (Figure 4A) and colony formation experiments (Figure 4B and C), silencing RIOK1 significantly inhibited glioma cell proliferation. Transwell migration and invasion assays further showed that knockdown significantly inhibited glioma cell migration and invasion abilities (Figure $4 \mathrm{D}$ and E). With regard to the molecular mechanism, 
western blot data showed that AKT1 and c-Myc protein levels were significantly downregulated following RIOK1 knockdown (Figure 4F and G).

\section{Discussion}

Glioma is derived from neural stromal cells including glial cells, ependymal cells, choroid plexus epithelial cells and neural parenchymal cells and is the most common primary tumor in central nervous system [18]. It has high incidence, high recurrence, and high mortality rates; the 5-year OS rate is only $13 \%$, and the median survival time is 14 months [19]. Our results demonstrate that RIOK1 and AKT1 expression are upregulated in glioma and correlate with poor prognosis.
A
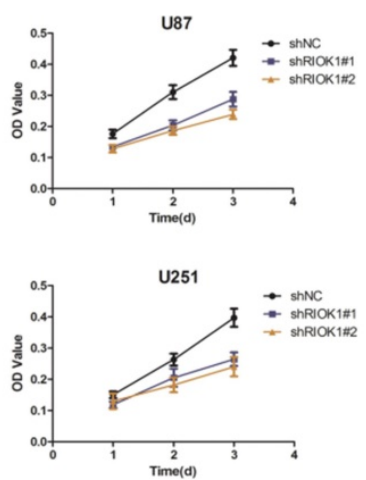

D
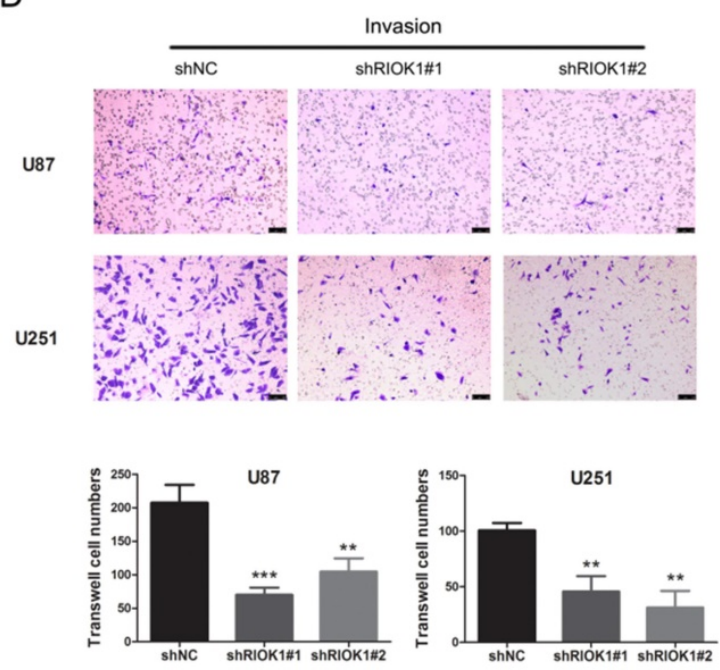

F

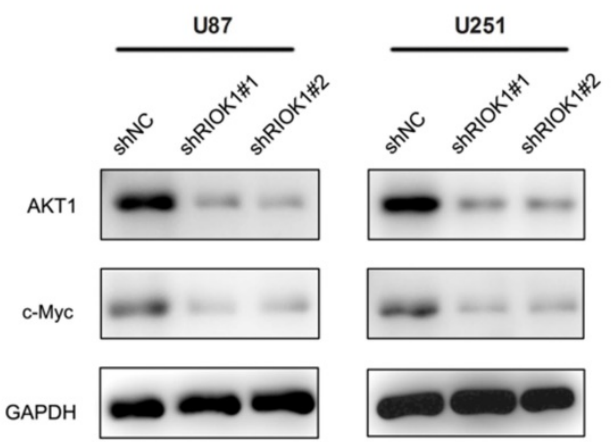

B
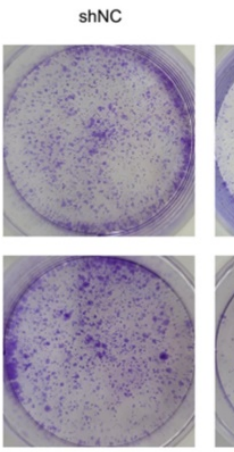

ShRIOK1\#1
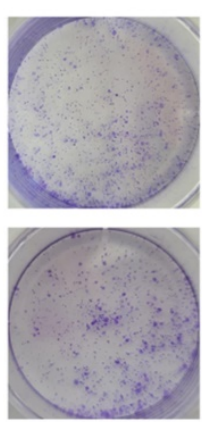

E

U87
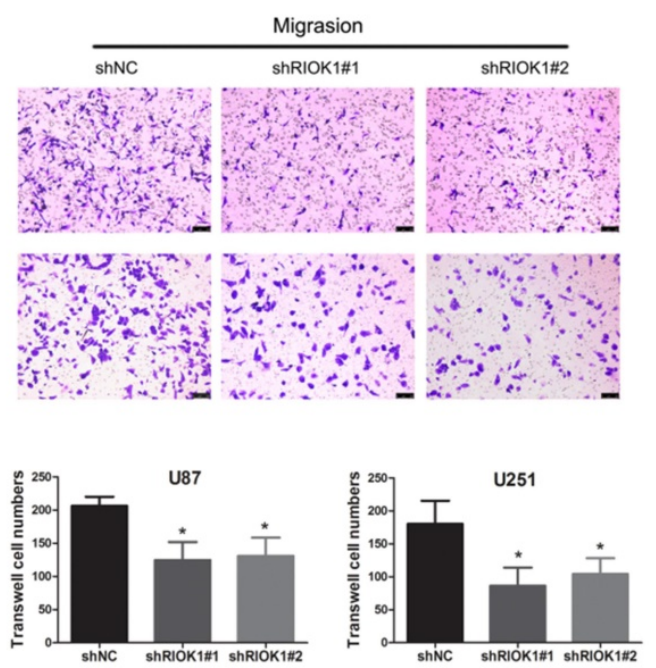

G
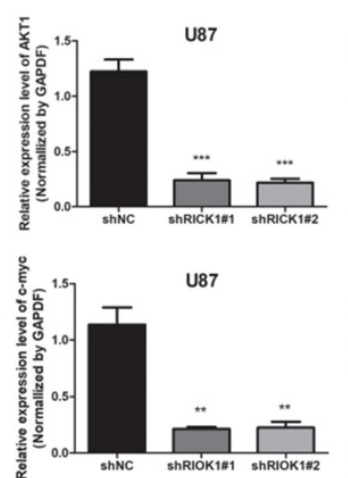

C
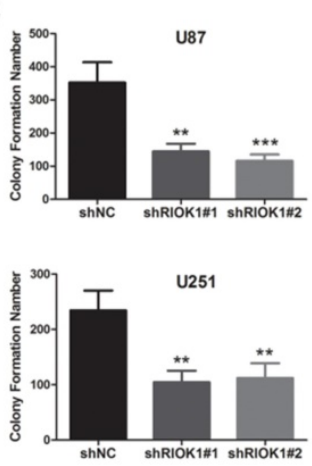
A member of the atypical kinase Rio family [20], RIOK1 is highly expressed in a variety of tumors and is involved in their malignant processes [21]. Although RIOK1 has been implicated in glioma cell proliferation and invasion, its expression, clinical significance and prognostic role in glioma tissue are still unknown. AKT1 is a crucial component of AKT signaling that contributes to the proliferation, migration, and invasion of multiple tumor types including glioma [22-27]. Increased AKT activity promotes glioma malignancy and cellular viability $[28,29]$. We investigated the co-expression of RIOK1 and AKT in GBM tissues and assessed its clinical significance. The tissue microarray results showed that RIOK1 and AKT1 expression in glioma tissues were significantly higher than those in matched normal brain tissues. In addition, tumors with higher WHO staging had the highest levels of RIOK1 and AKT1, and their expression was positively correlated. Finally, we found that both RIOK1 and AKT1 expression were correlated with poor prognosis.

Tumor cell proliferation and invasion are the main reasons leading to poor prognosis [30-33], so it is particularly important to explore possible molecular mechanisms. The human protein kinase RIOK1 is closely related to cell proliferation, and silencing RIOK1 causes cell cycle arrest [34]. Researchers also found that RIOK1 can interact with the Ras protein, which often contains mutations that promote tumor growth and metastasis [35]. In vitro studies have shown that the RIOK1-AKT signaling plays an important role in the malignant phenotype of glioma cells. Consistent with the previous studies, RIOK1 knockdown inhibited glioma cell proliferation, colony formation, migration, and invasion.

While investigating the molecular mechanism, we found that AKT1 and c-Myc expression were downregulated after silencing RIOK1. As a typical oncogenic gene, c-Myc participates in the regulation of pericellular adhesion [36], and its overexpression is closely related to the occurrence and metastasis of many kinds of tumors $[37,38]$. Since it is a transcription factor, c-Myc needs to enter the nucleus to regulate cell proliferation, apoptosis, and the cell cycle [39, 40]. Indeed, after RIOK1 knockout, c-Myc protein levels were significantly decreased, which inhibits glioma progress. These results indicate that RIOK1 can promote glioma cell progression by acting on c-Myc and AKT1.

In summary, our results provide a theoretical basis for the importance of the RIOK1-AKT1 axis in glioma occurrence and malignant progression. The RIOK1-AKT1 pathway has the potential to become a new target for the clinical diagnosis and treatment of gliomas.

\section{Abbreviations}

AKT1: protein kinase B; FBS: fetal bovine serum; GBM: glioblastoma multiforme; LGG: Low grade glioma; IHC: immunohistochemistry; mTOR: mammalian target of rapamycin; OS: overall survival; RIOK1: right open reading frame kinase 1; TCGA: The Cancer Genome Atlas; GTEx: Genotype-Tissue Expression; WHO: World Health Organization.

\section{Supplementary Material}

Supplementary figure.

http://www.jcancer.org/v12p5745s1.pdf

\section{Acknowledgements}

This study was supported by the Natural Science Foundation of Liaoning Province (grant number 20170520040), National Natural Science Foundation of China (grant number 81702738), Doctoral Startup Foundation of Liaoning Province (grant number 201501004), the Scientific Foundation of Liaoning Province (grant number SYYX202007), Scientific Foundation of Liaoning Province (grant number 310170007), and Natural Science Foundation of Liaoning Province (grant number 201602732).

\section{Ethics Committee Approval and Patient Consent}

The use of human glioma tissues in this study was approved by ethical committee of The First Affiliated Hospital of China Medical University at Shenyang. Written informed consent was obtained from all participants.

\section{Competing Interests}

The authors have declared that no competing interest exists.

\section{References}

1. Alexander BM, Cloughesy TF. Adult Glioblastoma. J Clin Oncol. 2017; 35: 2402-9.

2. Lah TT, Novak M, Breznik B. Brain malignancies: Glioblastoma and brain metastases. Semin Cancer Biol. 2020; 60: 262-73.

3. Buckner JC, Brown PD, O'Neill BP, Meyer FB, Wetmore CJ, Uhm JH. Central nervous system tumors. Mayo Clin Proc. 2007; 82: 1271-86.

4. Louis DN, Ohgaki H, Wiestler OD, Cavenee WK, Burger PC, Jouvet A, et al. The 2007 WHO classification of tumours of the central nervous system. Acta Neuropathol. 2007; 114: 97-109.

5. Gousias K, Markou M, Voulgaris S, Goussia A, Voulgari P, Bai M, et al. Descriptive epidemiology of cerebral gliomas in northwest Greece and study of potential predisposing factors, 2005-2007. Neuroepidemiology. 2009; 33: 89-95.

6. Stupp R, Hegi ME, Mason WP, van den Bent MJ, Taphoorn MJ, Janzer RC, et al. Effects of radiotherapy with concomitant and adjuvant temozolomide versus radiotherapy alone on survival in glioblastoma in a randomised phase III study: 5-year analysis of the EORTC-NCIC trial. Lancet Oncol. 2009; 10: 459-66.

7. Widmann B, Wandrey F, Badertscher L, Wyler E, Pfannstiel J, Zemp I, et al. The kinase activity of human Rio1 is required for final steps of cytoplasmic maturation of 40 S subunits. Mol Biol Cell. 2012; 23: 22-35.

8. Zemp I, Wild T, O'Donohue MF, Wandrey F, Widmann B, Gleizes PE, et al. Distinct cytoplasmic maturation steps of $40 \mathrm{~S}$ ribosomal subunit precursors require hRio2. J Cell Biol. 2009; 185: 1167-80. 
9. Weinberg F, Reischmann N, Fauth L, Taromi S, Mastroianni J, Kohler M, et al. The Atypical Kinase RIOK1 Promotes Tumor Growth and Invasive Behavior. EBioMedicine. 2017; 20: 79-97.

10. Hong $X$, Huang $H$, Qiu $X$, Ding $Z$, Feng $X$, Zhu $Y$, et al. Targeting posttranslational modifications of RIOK1 inhibits the progression of colorectal and gastric cancers. Elife. 2018; 7.

11. Zhang T, Ji D, Wang $\mathrm{P}$, Liang $\mathrm{D}$, Jin $\mathrm{L}$, Shi $\mathrm{H}$, et al. The atypical protein kinase RIOK3 contributes to glioma cell proliferation/survival, migration/invasion and the AKT/mTOR signaling pathway. Cancer Lett. 2018; 415: 151-63.

12. Read RD, Fenton TR, Gomez GG, Wykosky J, Vandenberg SR, Babic I, et al. A kinome-wide RNAi screen in Drosophila Glia reveals that the RIO kinases mediate cell proliferation and survival through TORC2-Akt signaling in glioblastoma. PLoS Genet. 2013; 9: e1003253.

13. Majewska E, Szeliga M. AKT/GSK3beta Signaling in Glioblastoma. Neurochem Res. 2017; 42: 918-24.

14. Chen Y, Huang L, Dong Y, Tao C, Zhang R, Shao H, et al. Effect of AKT1 (p. E17K) Hotspot Mutation on Malignant Tumorigenesis and Prognosis. Front Cell Dev Biol. 2020; 8: 573599.

15. Carpten JD, Faber AL, Horn C, Donoho GP, Briggs SL, Robbins CM, et al. A transforming mutation in the pleckstrin homology domain of AKT1 in cancer. Nature. 2007; 448: 439-44.

16. Tang Y, Wang R, Zhang Y, Lin S, Qiao N, Sun Z, et al. Co-Upregulation of 14-3-3zeta and P-Akt is Associated with Oncogenesis and Recurrence of Hepatocellular Carcinoma. Cell Physiol Biochem. 2018; 45: 1097-107.

17. Wang Y, Zang J, Zhang D, Sun Z, Qiu B, Wang X. KDM2B overexpression correlates with poor prognosis and regulates glioma cell growth. Onco Targets Ther. 2018; 11: 201-9.

18. Bush NA, Chang SM, Berger MS. Current and future strategies for treatment of glioma. Neurosurg Rev. 2017; 40: 1-14.

19. Molinaro AM, Taylor JW, Wiencke JK, Wrensch MR. Genetic and molecular epidemiology of adult diffuse glioma. Nat Rev Neurol. 2019; 15: 405-17.

20. Dez C, Tollervey D. Ribosome synthesis meets the cell cycle. Curr Opin Microbiol. 2004; 7: 631-7.

21. Berto G, Ferreira-Cerca S, De Wulf P. The Rio1 protein kinases/ATPases: conserved regulators of growth, division, and genomic stability. Curr Genet. 2019; 65: 457-66.

22. Li GQ, Zhang Y, Liu D, Qian YY, Zhang H, Guo SY, et al. PI3 kinase/Akt/HIF-1alpha pathway is associated with hypoxia-induced epithelial-mesenchymal transition in fibroblast-like synoviocytes of rheumatoid arthritis. Mol Cell Biochem. 2013; 372: 221-31.

23. Vivanco I, Sawyers CL. The phosphatidylinositol 3-Kinase AKT pathway in human cancer. Nat Rev Cancer. 2002; 2: 489-501.

24. Dillon RL, Muller WJ. Distinct biological roles for the akt family in mammary tumor progression. Cancer Res. 2010; 70: 4260-4.

25. Luo J, Manning BD, Cantley LC. Targeting the PI3K-Akt pathway in human cancer: rationale and promise. Cancer Cell. 2003; 4: 257-62.

26. Chautard E, Ouedraogo ZG, Biau J, Verrelle P. Role of Akt in human malignant glioma: from oncogenesis to tumor aggressiveness. J Neurooncol. 2014; 117: 205-15.

27. Phillips HS, Kharbanda S, Chen R, Forrest WF, Soriano RH, Wu TD, et al. Molecular subclasses of high-grade glioma predict prognosis, delineate a pattern of disease progression, and resemble stages in neurogenesis. Cancer Cell. 2006; 9: 157-73.

28. Wlodarski P, Grajkowska W, Lojek M, Rainko K, Jozwiak J. Activation of Akt and Erk pathways in medulloblastoma. Folia Neuropathol. 2006; 44: 214-20.

29. Schlegel J, Piontek G, Budde B, Neff F, Kraus A. The Akt/protein kinase B-dependent anti-apoptotic pathway and the mitogen-activated protein kinase cascade are alternatively activated in human glioblastoma multiforme. Cancer Lett. 2000; 158: 103-8.

30. Guo Q, Su N, Zhang J, Li X, Miao Z, Wang G, et al. PAK4 kinase-mediated SCG10 phosphorylation involved in gastric cancer metastasis. Oncogene. 2014; 33: 3277-87.

31. Klein CA. Cancer progression and the invisible phase of metastatic colonization. Nat Rev Cancer. 2020; 20: 681-94.

32. Achrol AS, Rennert RC, Anders C, Soffietti R, Ahluwalia MS, Nayak L, et al. Brain metastases. Nat Rev Dis Primers. 2019; 5: 5

33. Valastyan S, Weinberg RA. Tumor metastasis: molecular insights and evolving paradigms. Cell. 2011; 147: 275-92.

34. Angermayr M, Roidl A, Bandlow W. Yeast Rio1p is the founding member of a novel subfamily of protein serine kinases involved in the control of cell cycle progression. Mol Microbiol. 2002; 44: 309-24.

35. Cox AD, Fesik SW, Kimmelman AC, Luo J, Der CJ. Drugging the undruggable RAS: Mission possible? Nat Rev Drug Discov. 2014; 13: 828-51.

36. Huang H, Weng H, Zhou H, Qu L. Attacking c-Myc: targeted and combined therapies for cancer. Curr Pharm Des. 2014; 20: 6543-54.

37. Dang CV. MYC on the path to cancer. Cell. 2012; 149: 22-35.

38. Dejure FR, Eilers M. MYC and tumor metabolism: chicken and egg. EMBO J. 2017; 36: 3409-20.

39. Ashrafizadeh $\mathrm{M}$, Zarabi A, Hushmandi $\mathrm{K}$, Moghadam ER, Hashemi $\mathrm{F}$, Daneshi S, et al. C-Myc Signaling Pathway in Treatment and Prevention of Brain Tumors. Curr Cancer Drug Targets. 2021; 21: 2-20.

40. Stine ZE, Walton ZE, Altman BJ, Hsieh AL, Dang CV. MYC, Metabolism, and Cancer. Cancer Discov. 2015; 5: 1024-39. 